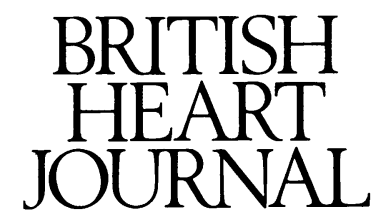

Editorial

\title{
Insulin and atherosclerosis: villain, accomplice, or innocent bystander?
}

Initial descriptions of an association between insulinaemia and both atherosclerotic cardiovascular disease and its risk factors appeared nearly 30 years ago. In 1969, Stout and Vallance-Owen suggested a direct role for insulin in atherogenesis. ${ }^{1}$ Stout recently summarised laboratory studies supporting a direct atherogenic effect of insulin through stimulation of vascular smooth muscle proliferation and arterial wall lipid deposition. ${ }^{2}$ Insulin has also been implicated as an indirect cause of atherogenesis through promoting the development of hypertension and dyslipidaemia. ${ }^{3-5}$

Several risk factors for atherosclerosis which cluster in individuals at high risk of atherosclerotic cardiovascular disease are associated with hyperinsulinaemia and/or insulin resistance. Reaven in 1988, postulated that insulin resistance and compensatory hyperinsulinaemia underlie this clustering. ${ }^{6} \mathrm{He}$ coined the term "syndrome X" (or the "insulin resistance syndrome") to describe the associations among insulin resistance, hyperinsulinaemia, glucose intolerance, dyslipidaemia, and hypertension, speculating that this syndrome may be an important cause of atherosclerotic cardiovascular disease in affluent societies.

Several mechanisms have been proposed for the association of insulin with atherosclerotic cardiovascular disease risk factor abnormalities. Insulin may contribute to the pathogenesis of hypertension by stimulating the sympathetic nervous system, promoting renal sodium retention, modulating cellular cation transport, and/or stimulating vascular smooth muscle hypertrophy. ${ }^{7}$ Insulin also may induce dyslipidaemia by stimulating hepatic synthesis of very low density lipoprotein (VLDL), leading to raised concentrations of triglyceride and depressed concentrations of high density lipoprotein (HDL) cholesterol. ${ }^{6}$

Indeed, hyperinsulinaemia is common in some populations with high rates of atherosclerotic cardiovascular disease such as Edinburgh men ${ }^{8}$ and immigrant Asian Indians. ${ }^{9}$ Three prospective population studies-the Helsinki Policemen Study, ${ }^{10}$ the Paris Prospective Study, ${ }^{11}$ and the Busselton Study ${ }^{12}$-have associated high insulin concentrations with an increased risk of atherosclerotic cardiovascular disease in men, but not in women (only the Busselton study included women). These studies have some limitations: HDL cholesterol was not measured and the findings were not consistent regarding a relation between atherosclerotic cardiovascular disease and fasting or post-glucose challenge insulin concentrations. In addition, a follow up of the Busselton study found that insulinaemia was an independent risk factor for total mortality in men, but not for cardiovascular mortality in either men or women. ${ }^{13}$ Insulin was not a risk factor for atherosclerotic cardiovascular disease in
Edinburgh or Gothenburg men. ${ }^{14} 15$ Pima Indians, who are markedly hyperinsulinaemic, have a low prevalence rate of atherosclerotic cardiovascular disease, and insulinaemia is not a predictor of ischaemic electrocardiographic abnormalities. ${ }^{16}$ Thus insulinaemia is not consistently associated with atherosclerotic cardiovascular disease.

The relation of insulin concentrations to atherosclerotic cardiovascular disease risk factors, especially hypertension, varies considerably. Nearly all studies showing a strong association between insulinaemia and blood pressure were conducted among groups of northern European origin. ${ }^{17-19}$ Studies in black subjects, ${ }^{19-21}$ Mexican-Americans, ${ }^{22}$ Nauruans, ${ }^{23}$ Pima Indians, ${ }^{19}$ and Asian Indians ${ }^{21}$ showed little or no association. Even among white subjects some data are discrepant. The CARDIA study showed comparable weak relations between blood pressure and fasting insulin concentrations among blacks and whites. ${ }^{20}$ The European Fat Distribution Study which was conducted among white women in Italy, the Netherlands, Sweden, and Poland found that diastolic blood pressure correlated with serum insulin in all countries except Sweden, whereas systolic blood pressure correlated with insulin only in Poland and southern Italy. After adjustment for body mass index, systolic and diastolic blood pressures were significantly correlated with insulinaemia only in subjects from southern Italy. ${ }^{24}$ Furthermore, Muller et $a l^{5}$ reported that insulinaemia accounted for less than $4 \%$ of the variance in blood pressure in an elderly white population in Baltimore. Although fewer data are available, associations between insulin concentrations and high concentrations of triglycerides and low concentrations of HDL cholesterol seem more consistent across populations ${ }^{45}$

The failure to find a consistent association between insulinaemia and atherosclerotic cardiovascular disease and some of its risk factors suggest that the relation is not causal or that the effects of insulin are primarily permissive. Insulin may act to accelerate atherogenesis in the presence of other risk factors such as hyperlipidaemia or hypertension. ${ }^{26}$ Modan et al reported that the excess risk of atherosclerotic cardiovascular disease found in hyperinsulinaemic men was confined to those who had at least one of three conditions: obesity, glucose intolerance, or hypertension. ${ }^{27}$ The Pima Indians, who are markedly hyperinsulinaemic but have low total cholesterol concentrations and a low prevalence of hypertension, have a low prevalence rate of atherosclerotic cardiovascular disease despite a considerably increased prevalence of noninsulin dependent diabetes. ${ }^{16}$ Limited evidence from clinical trials suggests that inducing hyperinsulinaemia may not have adverse effects on rates of atherosclerotic cardiovascular disease. The Coronary Drug Project showed that the hypolipidaemic drug nicotinic acid decreased total 
and cardiovascular mortality in men with previous myocardial infarction, ${ }^{28}$ though it causes insulin resistance and hyperinsulinaemia. ${ }^{29}$ The University Group Diabetes Program did not show any adverse effects of insulin treatment on cardiovascular complications. ${ }^{30}$

These observations and the stronger associations of insulin resistance than insulinaemia with blood pressure and lipid abnormalities in several studies suggest that insulin resistance rather than insulin may be the key factor with insulin as an innocent bystander merely reflecting insulin resistance. Several mechanisms have been proposed. Decreased insulin sensitivity in adipose tissue, especially visceral fat, can cause accelerated lipolysis and excessive delivery of free fatty acids to the liver resulting in increased VLDL production and hypertriglyceridaemia. Insulin resistance may reduce plasma triglyceride clearance (because of an associated reduction in lipoprotein lipase activity within the vasculature) further amplifying the increase in the concentrations of triglyceride rich lipoprotein. When the plasma residence time of these lipoproteins is increased they become cholesterol enriched and presumably more atherogenic, with reductions in HDL cholesterol concentrations. ${ }^{31}$ Insulin resistance could lead to the development of hypertension through blunting of insulin induced vasodilatation. ${ }^{32}$ Insulin resistance also is associated with increased concentrations of plasminogen activator inhibitor-1 which inhibits fibrinolysis. ${ }^{33}$ These changes could accelerate development of atherosclerosis and predispose to thrombosis, leading to an increased risk of atherosclerotic cardiovascular disease.

Alternatively, the association between hyperinsulinaemia or insulin resistance and atherosclerotic cardiovascular disease may not be causal but merely linked through common underlying factors. Both insulinaemia and insulin resistance are strongly correlated with adiposity, and much of the impact of insulin on risk of atherosclerotic cardiovascular disease could be related to amounts of body fat and its distribution. Increased sensitivity to glucocorticoids could produce an association. ${ }^{34}$ Glucocorticoids favour deposition of central fat and can induce insulin resistance, hypertension, and hyperlipidaemia. Increased activity of the sympathetic nervous system could lead to hypertension, insulin resistance, and hyperlipidaemia. ${ }^{19}$

Hyperinsulinaemia and insulin resistance are related to atherosclerotic cardiovascular disease and to the clustering of its risk factors in individuals. Their roles as independent atherosclerotic cardiovascular disease risk factors are, however, less certain. At present, it can be concluded that neither hyperinsulinaemia nor insulin resistance is a major risk factor for the development of atherosclerotic cardiovascular disease in the absence of other risk factors. Further laboratory research and prospective population studies of insulin resistance are needed to determine whether the observed relations are part of a causal chain for the development of atherosclerosis.

For now, what are the therapeutic implications of the insulin resistance syndrome? Should individuals be screened for hyperinsulinaemia or insulin resistance? Can either be modified? Should conventional drug regimens for treatment of diabetes and other risk factors for atherosclerotic cardiovascular disease be modified?

Practising physicians need to be aware of the interrelated features of the insulin resistance syndrome to avoid overlooking abnormalities that increase the risk of atherosclerotic cardiovascular disease. ${ }^{35}$ Routine determination of fasting insulin concentrations or attempts to measure insulin resistance are not justified. Though insulin resistance is partially genetically determined, it worsens with aging, weight gain, physical inactivity, and possibly a high fat diet. Maintenance of normal body weight is recommended. Obese people should be encouraged to lose weight and to maintain the weight loss because this will benefit all features of the insulin resistance syndrome. Exercise also is beneficial because it increases insulin sensitivity. ${ }^{36}$

Some have recommended avoiding use of diuretics and $\beta$ blockers in the treatment of hypertension, particularly in obese subjects at high risk of developing diabetes. The United States Joint National Committee on Detection, Evaluation, and Treatment of High Blood Pressure recommends these drugs still be used as initial therapy for hypertension, but points out that caution is needed with antihypertensive treatment in diabetics. ${ }^{37}$ Recent results from the Systolic Hypertension in the Elderly Study showed reduced incidence of stroke and coronary heart disease when hypertension was treated with these drugs. ${ }^{38}$ Therefore, a prudent course is to initiate antihypertensive therapy with a low dose of one of these drugs, monitor patients for significant shifts in risk factors for atherosclerostic cardiovascular disease, and use more expensive drugs only when they have specific advantages. In patients with non-insulin dependent diabetes mellitus there is no reason to withhold insulin treatment that is needed to control glycaemia. It is advisable, however, to emphasise the role of diet and exercise in treatment of non-insulin dependent diabetes mellitus and avoid inducing hyperinsulinaemia by overzealous use of long acting insulins or sulphonylureas. ${ }^{35}$

Preliminary data indicate that some drugs improve insulin sensitivity and further developments in this area may be of great importance in the treatment of both the insulin resistance syndrome and the prevention of noninsulin dependent diabetes mellitus. The biguanide metformin is useful for diabetic patients because it reduces glucose concentrations without increasing insulin secretion and may increase insulin sensitivity. ${ }^{39}$ It may have a role in subjects with impaired glucose tolerance and in normoglycaemic obese subjects. A new group of drugs that improve insulin sensitivity "thiozolidinoliones", is undergoing clinical trials to determine long-term safety and efficacy. ${ }^{40}$ In addition, patients with hyperlipidaemia and high concentrations of free fatty acids may benefit from the nicotinic acid analogue acipimox which inhibits lipolysis and improves insulin sensitivity. ${ }^{41}$ Though these new drugs seem promising, their ability to reduce the risk of atherosclerotic cardiovascular disease needs to be established by clinical trials. For now, diet and exercise remain the mainstays of treatment.

Clinical and Genetic Epidemiology Branch,

PETER J SAVAGE

Epidermiology and Biometry Program,

Division of Clinical Applications,

National Heart Lung and Blood Institute,

National Institute of Health,

Bethesda, Maryland, USA

Division of Diabetes,

MOHAMMED F SAAD

Department of Medicine,

School of Medicine,

University of Southern California,

Los Angeles, California, USA

Stout RW, Vallance-Owen J. Insulin and atheroma. Lancet 1969; i: $1078-80$.

2 Stout RW. Insulin and atheroma. 20-yr perspective. Diabetes Car 1990;13:631-54.

3 Modan M, Halkin H, Almog S, et al. Hyperinsulinemia: A link between Modan M, Halkin H, Almog S, et al. Hyperinsulinemia: A link between
hypertension, obesity and glucose intolerance. $f$ Clin Invest

4 Abbott WG, Lillioja S, Young AA, et al. Relationships between plasma lipoprotein concentrations and insulin action in an obese hyperinsulinemic population. Diabetes 1987;36:897-904. 
5 Zavaroni I, Bonora E, Pagliara M, et al. Risk factors for coronary artery disease in healthy persons with hyperinsulinemia and normal glucose tolerance. $N$ Engl $₹$ Med 1989;320:702-6.

6 Reaven GM. Role of insulin resistance in human disease. Diabetes 1988;37:1595-1607

7 DeFronzo RA, Ferrannini E. Insulin resistance: a multifaceted syndrome responsible for NIDD, obesity, hypertension, dyslipidemia, and atherosclerotic cardiovascular disease. Diabetes Care 1991;14:173-94

8 Logan RL, Reimesma RA, Thomson M, et al. Risk factors for ischaemic heart-disease in normal men aged 40: Edinburgh-Stockholm Study. Lancet 1978; i:949-52.

9 McKeigue PM, Shah B, Marmot MG. Relation of central obesity and insulin resistance with high diabetes prevalence and cardiovascular risk in South Asians. Lancet 1991;337:382-6.

10 Pyörälä K. Relationship of glucose tolerance and plasma insulin to the incidence of coronary heart disease: results from two population studies in Finland. Diabetes Care 1979;2:131-41.

11 Ducimentiere P, Eschwege R, Papoz L, et al. Relationship of plasma insulin level to the incidence of myocardial infarction and coronary heart disease. Diabetologia 1980;19:205-10.

12 Welborn TA, Wearne $\mathrm{K}$. Coronary heart disease incidence and cardiovascular mortality in Busselton with reference to glucose and insulin concentrations. Diabetes Care 1979;2:154-60.

13 Cullen K, Stenhouse NS, Wearne KI, et al. Multiple regression analysis of risk factors for cardiovascular disease and cancer mortality in Busselton, Western Australia-13-year study. F Chron Dis 1983;36: 371-7.

14 Hargreaves AD, Logan RL, Elton RA, et al. Glucose tolerance, plasma insulin, HDL cholesterol and obesity: 12-year follow-up and development of coronary heart disease in Edinburgh men. Atherosclerosis 1992;94:61-9.

15 Welin L, Eriksson H, Larsson B, et al. Hyperinsulinemia is not a major coronary risk factor in elderly men: The study of men born in 1913. Diabetologia 1992;35:766-70.

16 Liu QZ, Knowler WC, Nelson RG, et al. Insulin treatment, endogenous insulin concentration, and ECG abnormalities in diabetic Pima Indians: cross-sectional and prospective analyses. Diabetes 1992;41: 1141-50.

17 Ferrannini E, Buzzigoli G, Bonadonna $R$, et al. Insulin resistance in essential hypertension. N Engl F Med 1987;317:350-7.

18 Pollare $\mathrm{T}$, Lithell $\mathrm{H}$, Berne $\mathrm{C}$. Insulin resistance is a characteristic feature of primary hypertension independent of obesity. Metabolism 1990;39:167-74

19 Saad MF, Lillioja S, Nyomba BL, et al. Racial differences in the relation between blood pressure and insulin resistance. $N$ Engl $f$ Med 1991;324:733-9.

20 Manolio TA, Savage PJ, Burke GL, et al. Association of fasting insulin with blood pressure and lipids in young adults: The CARDIA Study Arteriosclerosis 1990;10:430-6.

21 Cruickshank JK, Cooper J, Burnett M, et al. Ethnic difference in fasting plasma C-peptide and insulin in relation to glucose tolerance and blood pressure, Lancet 1991;338:842-7.

22 Haffner SM, Fong D, Hazuda HP, et al. Hyperinsulinemia, upper body adiposity, and cardiovascular risk factors in non-diabetics. Metabolism 1988;37:338-45.
23 Collins VR, Dowse GK, Finch CF, et al. An inconsistent relationship between insulin and blood pressure in three Pacific island populations. between insulin and blood pressure

24 Cigolini M, Seidell JC, Charzewska J, et al. Fasting serum insulin in relation to fat distribution, serum lipid profile, and blood pressure in European women: The European Fat Distribution Study. Metabolism 1991;40:781-7.

25 Muller DC, Elahi D, Pratley RE, et al. An epidemiologic test of the hyperinsulinemia-hypertension hypothesis. $f$ Clin Endocrinol Metab 1993;76:544-8.

26 Durrington PN. Is insulin atherogenic? Diabetic Med 1992;9:597-600.

27 Modan M, Or J, Karaski A, et al. Hyperinsulinemia, sex, and risk of atherosclerotic cardiovascular disease. Circulation 1991;84:1165-75.

28 Canner PL, Berge KG, Wenger NK, et al. Fifteen year mortality in Coronary Drug Project patients: Long term benefit with niacin. $f A m$ Coll Cardiol 1986;8:1245-55.

29 Kahn SE, Beard JC, Schwartz MW, et al. Increased $\beta$-cell secretory capacity as mechanism for islet adaptation to nicotinic acid-induced insulin ity as mechanism for islet adaptation
resistance. Diabetes $1989 ; 38: 562-8$.

30 The University Group Diabetes Program. Effects of hypoglycemic agents on vascular complications in patients with adult-onset diabetes. Diabetes on vascular complicatio

31 Frayn KN, Coppack SW. Insulin resistance, adipose tissue and coronary heart disease. Clin Sci 1992;82:1-8.

32 Laakso M, Edelman SV, Brechtel G, et al. Decreased effect of insulin to stimulate skeletal muscle blood flow in obese men: a novel mechanism for insulin resistance. $\mathcal{F}$ Clin Invest 1990;85:1844-52.

33 Juhan-Vague I, Vague P. Hypofibrinolysis and insulin resistance. Diabetes Metab 1991;17:96-100.

34 Brindley DN, Rolland Y. Possible connections between stress, diabetes, obesity, hypertension and altered lipoprotein metabolism that may result in atherosclerosis. Clin Sci 1989;77:453-61.

35 Karam JH. Type II diabetes and syndrome X: pathogenesis and glycemic management. Endocrinol Metab Clin North Am 1992;21:329-50.

36 Eriksson KF, Lindgärde F, Prevention of type 2 (non-insulin-dependent) diabetes mellitus by diet and physical exercise. The 6-year Malmö feasidiabetes mellitus by diet and physical ex

37 National High Blood Pressure Education Program (US). The fifth report of the Joint National Committee on Detection, Evaluation, and Treatment of High Blood Pressure (JNCV). Arch Intern Med 1993; 153:149-52.

38 SHEP Cooperative Research Group. Prevention of stroke by antihypertensive drug treatment in older persons with isolated systolic hypertension. Final results of the Systolic Hypertension in the Elderly Program (SHEP). 尹AMA 1991;265:3255-64

39 Widén EIM, Eriksson JG, Groop LC. Metformin normalizes nonoxidative glucose metabolism in insulin-resistant normoglycemic first-degree relatives of patients with NIDDM. Diabetes 1992;41:354-8.

40 Suter SL, Nolan JJ, Wallace P, et al. Metabolic effects of a new oral hypoglycemic agent CS-045 in NIDDM subjects. Diabetes Care 1992;15:193-5.

41 Fulcher GR, Walker M, Catalano C, et al. Overnight suppression of lipolysis with acipimox increases carbohydrate oxidation and lowers fasting
blood glucose in type 2 (non-insulin dependent) diabetes [abstr]. Diabetologia 1989;32:489.
Diad 